\title{
Influencers e INDECOPI: el problema de la solución a la publicidad encubierta
}

Alejandro Díaz-Valdez

Abogado por la Pontificia Universidad Católica Argentina. Abogado por la Pontificia Universidad Católica del Perú. Máster en Comunicación Corporativa y Empresarial por la Universidad de Barcelona.

SUMARIO:

I. Introducción.

II. Marco normativo actual.

1. Ley de Represión de la Competencia Desleal.

2. Código de Protección y Defensa del Consumidor.

3. Leyes sectoriales.

III. El Proyecto.

1. Intención.

2. Problemas.

2.1. ¿Quién es un influencer?

2.2. ¿Qué implica el contacto previo del anunciante?

2.3. ¿Es suficiente el \#publicidadcontratada?

IV. Conclusiones. 


\section{RESUMEN:}

La llegada de los influencers ha generado una nueva forma de publicidad y es por eso que el Instituto Nacional de Defensa de la Competencia y de la Propiedad Intelectual-INDECOPI presentó la "Guía sobre Publicidad Digital y recomendaciones para Influencers [Proyecto]" con el objetivo de ofrecer lineamientos que deberían ser seguidos por influencers al publicar publicidad en sus redes sociales y no incumplir, entre otros, la Ley de Represión de la Competencia Desleal. Este artículo busca presentar al lector los principales problemas que se articulan de la lectura del proyecto de guía mencionada y las dificultades que su redacción actual supone para su ejecución.

Palabras clave: influencers, publicidad, INDECOPI, competencia, redes sociales.

\section{ABSTRACT:}

The arrival of influencers has generated a new type of advertising, which is why the Peruvian National Institute for the Defense of Competition and Intellectual Property-INDECOPI presented the "Guide on Digital Advertising and recommendations for Influencers [Project]". It offers guidelines that influencers should follow when posting advertisement in their social media and, in that way, not fail to comply with, among others, the Repression of Unfair Competition Law. This article aims to present to the reader the main problems that arise from the reading of the mentioned project guide and the difficulties its current wording poses for its execution.

Keywords: influencers, advertising, INDECOPI, Competition Law, social media.

\section{INTRODUCCIÓN}

Los avances tecnológicos en las formas de comunicación han originado la aparición nuevos puntos de encuentro entre anunciantes, oferentes y consumidores y la forma de interactuar entre ellos. Internet, y más específicamente las redes sociales, han propiciado el advenimiento de espacios novedosos donde las empresas pueden encontrar a su público y así potenciar su presencia de marca y la comercialización de sus productos.

Sin embargo, si no fuera por la aparición de ciertos personajes, la relación entre anunciantes, oferentes y consumidores y la forma en la cual los productos y/o servicios son presentados a los potenciales consumidores seguiría siendo la misma a través del tiempo: el anunciante intentando acercar un producto o servicio a un consumidor dispuesto a adquirirlo. En internet, por ejemplo, a través de banners y/o videos. Tradicionalmente, un anunciante se serviría de una figura pública para conectar sus productos y/o servicios con su público objetivo considerando la imagen que quisiera proyectar en sus potenciales consumidores. Dicha figura pública podría, justamente, ser pública en virtud de su profesión -actor, deportista, músico, cantante, chef, modelo, etc.-, pero difícilmente en virtud de la confianza y capacidad de influenciar la toma de decisiones de potenciales consumidores.

La llegada de los influencers como generadores de opinión e "influenciadores" de comportamiento ha generado una nueva forma de conectar anunciantes y oferentes con sus consumidores. Éstos, basados en la credibilidad que sus seguidores - potenciales consumidoreshan depositado en aquellos, los eligen para publicitar sus productos $\mathrm{y} / \mathrm{o}$ servicios y acercarse a su público objetivo de una manera más natural y espontánea.

Sin embargo, la línea que determina qué contenido es propio y espontáneo de un influencer y cuál es un contenido pagado por y realizado en beneficio de un anunciante es, cuanto menos, difusa. Es por eso que el Instituto Nacional de Defensa de la Competencia y de la Propiedad Intelectual-INDECOPI presentó, en el último cuatrimestre de 2019, la "Guía sobre Publicidad Digital y recomendaciones para Influencers [Proyecto]"

1. Guía sobre Publicidad Digital y recomendaciones para Influencers [Proyecto], de 2019, publicado por INDECOPI, https:// www.indecopi.gob.pe/documents/20182/3979412/Publicidad+Digital.pdf/186e5bc2-42c6-9a30-1d5c-dff598e82358. 
con el objetivo de ofrecer los lineamientos que deberían ser seguidos por los influencers para publicar publicidad en sus redes sociales -en adelante, el "Proyecto"—.

La intención de este artículo es presentar al lector los principales problemas que se articulan de la lectura del Proyecto y las dificultades que su redacción actual supone para su ejecución.

\section{MARCO NORMATIVO ACTUAL}

En la actualidad, existen dos grandes cuerpos normativos que regulan las obligaciones y las consecuencias relacionadas con la publicidad de productos y/o servicios: Ley de Represión de la Competencia Desleal y Código de Protección al Consumidor.

\section{Ley de Represión de la Competencia Des-} leal.

La Ley de Represión de la Competencia Desleal "(...) reprime todo acto o conducta de competencia desleal que tenga por efecto, real o potencial, afectar o impedir el adecuado funcionamiento del proceso competitivo²." En concreto, la Ley de Represión de la Competencia Desleal busca proteger a los partícipes del mercado - sean anunciantes, oferentes, consumidores, medios de comunicación social, etc.- de prácticas desleales y/o anticompetitivas que, en última instancia, podrían afectar negativamente la conducta de un consumidor.

El Proyecto indica que, entre otras cuestiones, la publicidad de influencers podría afectar los siguientes principios contenidos en la Ley de Represión de la Competencia Desleal:

a) Actos de engaño - artículo 8-33: son actos que afectan la transparencia del mercado $y$, en líneas generales, se relacionan con la veracidad de las características y condiciones informadas al mercado sobre ciertos productos y/o servicios.

b) Actos contra el principio de autenticidad —artículo 16- ${ }^{4}$ : son actos que impiden al consumidor reconocer el contenido publicitario como tal. Este tipo de actividades, en el caso de los influencers, es justa-

2. Artículo 1 del Decreto Legislativo 1044, de 25 de junio de 2008, por el que se aprueba la Ley de Represión de la Competencia Desleal.

3. Artículo 8: Actos de engaño:

8.1.- Consisten en la realización de actos que tengan como efecto, real o potencial, inducir a error a otros agentes en el mercado sobre la naturaleza, modo de fabricación o distribución, características, aptitud para el uso, calidad, cantidad, precio, condiciones de venta o adquisición y, en general, sobre los atributos, beneficios o condiciones que corresponden a los bienes, servicios, establecimientos o transacciones que el agente económico que desarrolla tales actos pone a disposición en el mercado; o, inducir a error sobre los atributos que posee dicho agente, incluido todo aquello que representa su actividad empresarial.

8.2.- Configuran actos de engaño la difusión de publicidad testimonial no sustentada en experiencias auténticas y recientes de un testigo.

8.3.- La carga de acreditar la veracidad y exactitud de las afirmaciones objetivas sobre los bienes o servicios anunciados corresponde a quien las haya comunicado en su calidad de anunciante.

8.4.- En particular, para la difusión de cualquier mensaje referido a características comprobables de un bien o un servicio anunciado, el anunciante debe contar previamente con las pruebas que sustenten la veracidad de dicho mensaje.

4. Artículo 16: Actos contra el principio de autenticidad:

16.1.- Consisten en la realización de actos que tengan como efecto, real o potencial, impedir que el destinatario de la publicidad la reconozca claramente como tal.

16.2.-Constituye una inobservancia a este principio difundir publicidad encubierta bajo la apariencia de noticias, opiniones periodísticas o material recreativo, sin advertir de manera clara su naturaleza publicitaria. Es decir, sin consignar expresa y destacadamente que se trata de un publirreportaje o un anuncio contratado. 
mente la razón por la cual el Proyecto ha sido concebido. En el caso de los influencers, el discernimiento entre qué contenido es propio, espontáneo y generado por el influencer y cuál contratado por un anunciante puede ser muy difícil de diferenciar.

\section{Código de Protección y Defensa del Con- sumidor.}

\section{El Código de Protección del Consumidor:}

"(...) tiene la finalidad de que los consumidores accedan a productos y servicios idóneos y que gocen de los derechos y los mecanismos efectivos para su protección, reduciendo la asimetría informativa, corrigiendo, previniendo o eliminando las conductas y prácticas que afecten sus legítimos intereses. En el régimen de economía social de mercado establecido por la Constitución, la protección se interpreta en el sentido más favorable al consumidor, de acuerdo a lo establecido en el presente Código."

Es decir, protege al consumidor por la diferencia de información que existe entre él y el proveedor $y / u$ oferente de un producto y/o servicio que lo pone a su disposición.

El Código de Protección y Defensa del Consumidor aplicará de manera supletoria a la Ley de Represión de la Competencia Desleal en la medida que la publicidad no constituya una oferta. En dicho caso, la oferta también estará protegida por el Código de Protección y Defensa del Consumidor. Al respecto, si bien la publicidad y la oferta tienen como objetivo que, eventualmente, un consumidor adquiera $\mathrm{y} / \mathrm{o}$ contrate los productos $y / o$ servicios ofrecidos por el anunciante, una publicidad es una comunicación de los atributos de un producto y/o servicio determinado, mientras que una oferta es una invitación a contratar bajo en los términos y condiciones ofrecidos.

\section{Normas sectoriales.}

Existen normas que regulan la forma en la que ciertos productos deben ser publicitados, como en el caso del tabaco, alcohol, medicamentos, entre otros. En estos supuestos la publicidad no sólo se regula de acuerdo a los principios generales establecidos en la Ley de Represión de la Competencia Desleal, sino que debe cumplir con los requerimientos específicos de dicha norma sectorial.

Así, por ejemplo, la Ley 28705 para la Prevención y Control de los Riesgos del Consumo del Tabaco establece, en su artículo 13, que los anuncios de productos de tabaco deberán consignar la frase "Prohibida su Venta a Menores de 18 Años"; la Ley 28861 que Regula la Comercialización y Consumo y Publicidad de Bebidas Alcohólicas establece, en su artículo 8, que la publicidad de toda bebida alcohólica deberá consignar la frase "Tomar bebidas alcohólicas en exceso es dañino".

En efecto, el artículo 17, inciso 2, de la Ley de Represión de la Competencia Desleal ${ }^{6}$, establece que será un incumplimiento a dicha ley, el incumplimiento de las normas sectoriales.

\section{EL PROYECTO}

\section{Intención.}

El Proyecto publicado por INDECOPI, si bien no constituye un marco normativo con carácter de ley exigible con pena de ser sancionado por incumplimiento, busca dar el puntapié inicial contra la publicidad encubierta que podrían realizar los influencers.

Asimismo, es importante remarcar desde el

5. Artículo 1 de la Ley 29571, de 1 de setiembre de 2010, Código de Protección y Defensa del Consumidor.

6. 17.2.- Constituye una inobservancia de este principio el incumplimiento de cualquier disposición sectorial que regule la realización de la actividad publicitaria respecto de su contenido, difusión o alcance. 
inicio que los influencers, en la medida que no publiciten productos $\mathrm{y} / \mathrm{o}$ servicios propios eventos, marcas de ropa, líneas de productos de cuidado personal y/o belleza, etc.- , son el medio de comunicación elegido por la anunciante $y$, por ende, no son anunciantes per se y no son responsables por el contenido de la publicidad.

En ese sentido, el Proyecto indica que "(...) la problemática gira en torno al uso de formato publicitarios que se utilizan en el marketing de influencers que no permitan los seguidores (consumidores) detectar con claridad cuando están frente a contenido publicitario o cuando es un mensaje auténtico del influencer (...)".

Es por ello que el Proyecto, sin constituir un cuerpo normativo independiente $y$ exigible per se, tiene como intención establecer ciertas recomendaciones y buenas prácticas para que los influencers publiquen contenido publicitario sin que supongan incumplimientos a la Ley de Represión de la Competencia Desleal. Así, la intención del Proyecto es determinar las situaciones en las cuales el influencer está haciendo publicidad de un producto y/o servicio, a los efectos de mantener correctamente informado a sus seguidores del carácter publicitario de dicho contenido.

\section{Problemas.}

El Proyecto, sin embargo, no es perfecto y su principal falla radica en la dificultad que se ha planteado para su aplicación, tanto en la definición de su ámbito de aplicación subjetivo quién-, como objetivo - cuándo-.

Al respecto, el Proyecto indica que "(...) se entenderá como publicidad aquellas prácticas realizadas por el influencer que promuevan, directa o indirectamente las marcas, productos o servicios del anunciante, siempre y cuando se hayan originado como consecuencia del contacto previo del anunciante a cambio de un pago o beneficio de cualquier tipo." ${ }^{7}$
Para entender a quién y cuándo aplicaría lo dispuesto en el Proyecto, resulta necesario desmenuzar su ámbito de aplicación:

\section{1. ¿Quién es un influencer?}

Si bien no es una novedad que los anunciantes se sirvan de figuras públicas para publicitar sus productos y/o servicios, la figura del influencer es relativamente reciente.

El Proyecto define a los influencers como:

"(...) una persona que cuenta con cierta credibilidad sobre un tema determinado y que, dada su capacidad de convencimiento o llegada a sus seguidores, sin tener en consideración necesariamente el número de estos, puede convertirse en un componente determinante en las decisiones que los consumidores realizan en el mercado."

A diferencia de una figura pública en razón de su profesión -actor, músico, cantante, chef, modelo, etc.-, un influencer genera en sus seguidores la sensación de cercanía y conocimiento, basada en algún tipo de atributo del influencer. El atributo que hace que un influencer sea tal es tan variado como influencers hay en el mundo, es decir, la relación con el seguidor puede ser en virtud del conocimiento de un tema específico, humor, éxito profesional, gusto determinado en ropa, gastronomía, música, etc.

Ahora bien, el primer obstáculo generado por el Proyecto está directamente relacionado, justamente, por quiénes calificarían como influencers. La definición otorgada por el Proyecto podría implicar que ninguno, o todos, los usuarios de redes sociales sean catalogados como influencers por carecer de criterios objetivos para su delimitación.

En efecto, ¿qué implica que una persona tenga "cierta credibilidad sobre un tema determinado"? 
Muchos influencers generan conexión con su público por ser referentes en temas específicos - moda o gastronomía, por ejemplo-, pero muchos otros no necesariamente son expertos en uno, o ningún, tema específico y simplemente han amasado una gran cantidad de seguidores en base a la forma en que se desenvuelven e interactúan con su público. Así, por ejemplo, ¿sería de aplicación lo detallado en el Proyecto para influencers que no son expertos en ningún tema? El hecho de gozar, o no, de credibilidad sobre un tema específico no necesariamente es un factor determinante para identificar a un influencer. No sólo no es un factor determinante, sino que la carga de la prueba relacionado a este componente es sumamente difícil, sino imposible de probar.

Así mismo, cabe preguntarse cómo haría el INDECOPI para probar que el contenido publicado por un influencer es, o ha sido, "un componente determinante en las decisiones que los consumidores realizan en el mercado". No cabe duda de que el término influencer se relaciona justamente con el hecho de "influenciar" un comportamiento, pero resultaría interesante conocer el mecanismo a implementar por el INDECOPI para determinar cuándo un influencer determinado efectivamente (a) ha influido en el comportamiento de sus seguidores a nivel general para ser catalogado como "influencer"; y (b) que la decisión de consumo del seguidor se ha dado por el hecho de haber sido influenciado por el influencer. Es decir, ¿cómo haría el INDECOPI para determinar que cierto usuario es considerado influencer por sobre otro basado en la capacidad de influenciar sobre decisiones de consumo de terceros? ¿Acaso utilizarán recursos para analizar hábitos de consumo de todos los usuarios de las redes para determinar si sus decisiones de consumo fueron influenciadas por otros usuarios? $\mathrm{Si}$ es así, ¿a cuántos usuarios más debería haber influenciado para ser considerado influencer? Difícilmente podría decirse que haber influenciado a una sola persona implique ser catalogado como un influenciador capaz de ser un "un componente determinante en las decisiones que los consumidores realizan en el mercado". ¿Cuál es el umbral que debería cruzarse para considerar a un usuario de redes sociales como "influencer"?

Es decir, para catalogar a un usuario de redes sociales como influencer sí es necesario considerar la cantidad de seguidores que éstos tienen en sus canales. Para aclarar, supongamos el siguiente ejemplo: la marca de licores espirituosos importados de Europa "XYZ" decide comenzar a comercializar sus productos en el Perú $y$, como estrategia de posicionamiento, decide contactarse con jóvenes de entre 28 y 35 años de los barrios más selectos de Lima para que publiquen contenido publicitario en sus redes sociales. Ninguno de los jóvenes seleccionados se considera, ni son considerados por sus pares, amigos y conocidos como influencers; simplemente gozan de una vida social activa $y$, por ende, tienen algunos pocos miles de seguidores en sus redes sociales. En concreto, ninguno de ellos cuenta con más de 2,500 seguidores Instagram. XYZ ofrece a estos jóvenes (a) solventar los gastos de una fiesta en sus casas, en donde se preparen tragos con el licor "XYZ"; $y$ (b) regalarle a cada uno de ellos una caja con 12 botellas de "XYZ", a cambio de la publicación de una serie de fotos, videos y stories en Instagram. ¿Califican los jóvenes como "influencers"? ¿Deberían indicar que se trata de \#publicidadcontratada? ¿Debería quedar englobado en el derecho de libre expresión? Es decir, los supuestos objetivos se habrían cumplido - contacto previo del anunciante y contraprestación - pero, ¿estos jóvenes podrían ser pasibles de sanciones administrativas por no indicar que su contenido era publicidad contratada? ¿Podría defenderse que ellos son influencers?

La definición de quién podría ser catalogado como influencer ofrecida por el Proyecto, si bien podría ser correcta en la teoría, resulta gaseosa y difusa en la práctica si se la quiere utilizar como parámetro para determinar quiénes estarían sujetos al cumplimiento de la normativa aplicable en materia publicitaria. Los componentes principales que sirven para determinar cuándo estamos frente a un influencer $y$, por ende, cuándo tales personas estarían obligadas a informar a sus seguidores que su contenido es publicidad contratada, son completamen- 
te subjetivos. No existen umbrales objetivos que permitan discernir con claridad cuándo un usuario de redes sociales es influencer o no, sino mecanismos de difícil comprobación basados en (a) conocimientos sobre un tema específico; y/o (b) la capacidad de influenciar el comportamiento de sus seguidores.

\section{2. ¿Qué implica el contacto previo del anun- ciante?}

El Proyecto establece que para que un contenido publicitario contratado sea tal, debe haber existido un contacto previo del anunciante al influencer. Esto, al igual que la definición de influencer que comentamos en la sección anterior, trae aparejado una serie de problemas.

Para comenzar, cabe preguntarse cuál es la herramienta que planea usar el INDECOPI para fiscalizar que esto efectivamente haya sido así. Considerando el principio de legalidad, que a grandísimos rasgos indica que los actos prohibidos están tipificados en las leyes que regulan su ejercicio y que para gatillar la sanción que traen aparejados es necesario que se cumplan tal como están redactados, el hecho de limitar el ámbito de aplicación a los casos en los cuales el influencer es contactado previamente por el anunciante impone una carga probatoria especialmente difícil para quien alegue que un anunciante ha incumplido con la normativa aplicable en materia publicitaria $y$, por ende, pasible de una sanción administrativa.

¿Qué significa un contacto previo? ¿En qué momento se gatilla dicho contacto previo? ¿Cómo puede la parte afectada probar que el influencer actuó bajo un contacto previo del anunciante? Un contrato posterior a la publicidad, por ejemplo, no serviría para probar el contacto previo; el contrato es la foto final, el resultado, de una negociación que debe ser iniciada por alguna de las partes. En ese escenario, ¿sería necesario tener que recurrir a la etapa precontractual e indagar cómo fue iniciada la negociación? Además, suponiendo que la intención del INDECOPI fuera efectivamente investigar la etapa precontractual del acuerdo entre el anunciante y el influencer, ¿cómo haría para determinar con certeza que el anunciante realizó el primer contacto previo con el influencer? Para quien haya participado en más de una negociación contractual, nunca es claro quién dio el puntapié inicial en las negociaciones.

Además, no existe razón alguna para excluir del ámbito de aplicación que el anunciante sea contactado previamente por el influencer, si también puede generarse contenido publicitario de esta manera. Es decir, si la intención del INDECOPI es evitar que los seguidores de los influencers carezcan de información certera en relación a potenciales publicidades encubiertas, entonces la etapa precontractual y la forma en la cual se generó el negocio jurídico entre influencer y anunciante debería serle completamente indiferente al INDECOPI. Si sirve de algo haber excluido la situación donde el influencer es quien contacta al anunciante es, justamente, para eludir responsabilidad por incumplimiento al Proyecto.

Así, la circunscripción del ámbito de aplicación al hecho de que el influencer haya sido previamente contactado por el anunciante no sólo parece arbitraria, sino que impone una carga de la prueba sumamente complicada para quien intente una acción administrativa contra un influencer por un supuesto de publicidad encubierta que, en la práctica, atenta contra el mismo principio de protección al consumidor que tanto busca proteger.

\section{3. ¿Es suficiente el \#publicidadcontratada?}

Finalmente, el Proyecto establece que:

"(...) los casos en los cuales no sea evidente para el consumidor que está frente a contenido publicitario o se tienen dudas de si es suficiente o no lo comunicado para que el consumidor interiorice dicha naturaleza de la publicación, el influencer debe expresar que su publicación es un anuncio contratado. Una opción para realizar esta comunicación es mediante el uso de hashtags como \#publicidad o \#publicidadcontratada."

La inclusión del hashtag, sin embargo, queda a

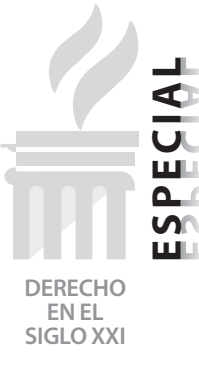


criterio del influencer basado en lo que él cree que podría ser, o no, un contenido publicitario evidente para sus seguidores.

Esto es, en esencia, un problema para los influencers dada la conexión que éstos tienen con sus seguidores. Como el mismo Proyecto señala, la interacción que los influencers tienen con sus seguidores es menos forzada e invasiva dado el enfoque natural y espontáneo que transmite el influencer ${ }^{8}$. Lo anterior, sumado a que queda a consideración del influencer determinar cuándo un contenido publicitario podría no ser evidente para sus seguidores, tiene como consecuencia un incentivo vil de, justamente, no indicar cuándo sus seguidores están frente a contenido publicitario contratado. Considerando que la conexión entre seguidores e influencers se da por el carácter espontáneo de su contenido, la publicación de contenido publicitario contratado atentaría contra la relación generada y podría ser un desincentivo para que el influencer informe que se trata de publicidad contratada.

De cualquier manera, el influencer podría sortear fácilmente esta obligación de información. $\mathrm{Si}$ el contenido publicado no califica como "publicidad", por carecer de cualquiera de los componentes descritos en los apartados 2.2 y 2.3 anteriores, entonces el influencer no estaría obligado a informar a sus seguidores que el contenido publicado es una publicidad contratada, contrariando, justamente, lo que el Proyecto busca proteger.

\section{CONCLUSIONES}

Es entendible que la punición de una conducta deba estar bien delimitada y sus alcances, bien descritos, de otra manera cualquier acto de la vida civil podría ser interpretado como una conducta subsumible dentro cualquier figura prohibida. Sin embargo, la descripción de la conducta prohibida deber ser lo suficiente- mente objetiva como para poder, efectivamente, proteger a terceros de la consumación de tal conducta. Ese es el principal problema del Proyecto.

Su intención es loable y deseada por el mercado; las recomendaciones para evitar que ninguna persona esté expuesta a publicidades encubiertas son más que bienvenidas, pero para que esta protección sea llevada a la práctica, es necesario que las conductas que configuran un incumplimiento tengan criterios objetivos. El hecho de que no haya criterios objetivos para determinar, en primera instancia, quién calificaría como influencer, es un obstáculo que dificulta a priori su implementación. Basar la aplicación de eventual sanciones en personas que (a) tengan conocimientos sobre un tema específico; y/o (b) la capacidad de influenciar el comportamiento de sus seguidores es, como mínimo, difícil de probar. Si habiendo podido probar con éxito la calidad de "influencer" de un usuario de redes sociales, aun así, nos toparemos con la también difícil tarea de probar que el contenido publicitario se dio en virtud de un contacto previo del anunciante.

Esto es más evidente aún si tomamos en consideración que las conductas sancionables prescinden de toda culpa o dolo para la atribución de responsabilidad. Si no existe un criterio de atribución subjetivo para una conducta determinada, es decir, si es indiferente si el denunciado ha tenido la intención de cometer la infracción — sumado a que también es indiferente que haya existido un daño, el simple hecho de haberse configurado el cumplimiento implica su sanción-, con más razón la descripción de la conducta prohibida debería proteger los intereses de terceros mediante una tipificación objetiva, clara y sin grises.

En efecto, el Proyecto parecería ser un buen consejo sin destinatario. La guía de recomenda-

8. Guía sobre Publicidad Digital y recomendaciones para Influencers [Proyecto], de 2019, publicado por INDECOPI, 21, acceso el 28 de noviembre de 2019, https://www.indecopi.gob.pe/documents/20182/3979412/Publicidad+Digital.pdf/186e5bc2-42c6-9a30-1d5c-dff598e82358. 
ciones sobre cómo publicar el contenido publicitario contratado carece de sentido si no existe claridad en cuanto a quién y cuándo debería seguir dichas recomendaciones para no ser sancionado por cumplimientos a la normativa aplicable en materia publicitaria.

Como dijimos anteriormente, cualquier, o ningún, usuario de redes sociales podrían ser catalogados como influencers por carecer de criterios objetivos para su delimitación.

No es intención de este artículo fijar los criterios objetivos necesarios para que las recomendaciones del Proyecto sean efectivas, sino plantear una discusión sobre cómo debería abordarse esta situación. Es evidente que el contenido publicitario contratado debe ser fácilmente identificable para usuarios de redes sociales, de manera que éstos puedan tomar las decisiones de consumo de la forma más informada posible. El Proyecto es el puntapié inicial en este sentido, pero resulta necesario continuar el debate entre influencers y las autoridades pertinentes a fin de acordar un texto que permita cumplir el fin buscado por la norma. 